Bull. Korean Math. Soc. 45 (2008), No. 4, pp. 709-715

\title{
ON SOME RING CLASS FIELDS BY SHIMURA'S CANONICAL MODELS
}

\author{
SoYoung Choi And Ja Kyung Koo
}

\begin{abstract}
We construct certain ring class fields over an imaginary quadratic field by making use of Shimura's canonical models and extend the result of Chen-Yui ([1] Theorem 3.7.5(2)) to the case where $(a, b, N) \neq N$ or $(a / N, N) \neq 1$ for a positive integer $N>1$.
\end{abstract}

\section{Introduction}

When $\Gamma_{0}(N)$ is the Hecke subgroup of $S L_{2}(\mathbb{Z})$ for a positive integer $N$, Helling showed in [3] that the group $\Gamma_{0}(N)^{*}$ generated by $\left\{\Gamma_{0}(N),\left(\begin{array}{cc}0 & -1 \\ N & 0\end{array}\right)\right\}$ has the genus zero exactly for $N=1 \sim 21,23 \sim 27,29,31,32,35,36$, $39,41,47,49,50,59,71$. Moreover, for all such $N$ but 49 and $50, \Gamma_{0}(N)^{*}$ has a fundamental Thompson series $T_{N}^{*}$ corresponding to itself ([2] Table 2). Throughout this paper, we denote $\alpha$ to be a Heegner point, that is, $\alpha$ is a root in $\mathfrak{H}$ of an integral equation $a z^{2}+b z+c=0$ with $b^{2}-4 a c<0,(a, b, c)=1$ and $a>0$, and $K$ to be an imaginary quadratic field $\mathbb{Q}(\alpha)$. Chen and Yui showed in [1] Theorem 3.7.5(2) by using the Shimura reciprocity law that when $(a, N)=1$ for a prime number $N, T_{N}^{*}(\alpha)$ generates a ring class field over $K$ of an imaginary quadratic order $\mathcal{O}^{\prime}$ of discriminant $f^{2} d_{K}$ where $f=m N$ and $b^{2}-4 a c=m^{2} d_{K}<0$.

In this paper, over an imaginary quadratic field $K$ we study the class fields generated by the singular values of automorphic functions which give rise to Shimura's canonical models of $\Gamma_{0}(N) \backslash \mathfrak{H}^{*}$ or $\Gamma_{0}(N)^{*} \backslash \mathfrak{H}^{*}$ at imaginary quadratic arguments in the complex upper half plane $\mathfrak{H}$ (Theorem 2.4). Here, $N$ is any positive integer and $\mathfrak{H}^{*}=\mathfrak{H} \cup \mathbb{P}^{1}(\mathbb{Q})$, and our result is independent of the genus of curve under consideration. As a corollary (Corollary 2.5), we can extend Chen-Yui's result on a ring class field $K\left(T_{N}^{*}(\alpha)\right)$ to the case where $(a, b, N) \neq N$ or $\left(\frac{a}{N}, N\right) \neq 1$ for a positive integer $N>1$ by using the theory of complex multiplication, whose proof is different from their argument.

Throughout the article we adopt the following notations:

Received February 11, 2008.

2000 Mathematics Subject Classification. 11F06, 20H10, 22E40, 30F35.

Key words and phrases. class fields, Shimura's canonical models, Thompson series.

This work was supported by Korea Research Foundation Grant(KRF-2002-070-C00003). 
- $\Gamma_{0}(N)=\left\{\left(\begin{array}{ll}a & b \\ c & d\end{array}\right) \in S L_{2}(\mathbb{Z}) \mid c \equiv 0 \bmod N\right\}$

- $\Gamma_{0}(N)^{*}=\left\langle\left\{\Gamma_{0}(N),\left(\begin{array}{cc}0 & -1 \\ N & 0\end{array}\right)\right\}\right\rangle$

- $\mathbb{Z}_{p}$ the ring of $p$-adic integers

- $\mathbb{Q}_{p}$ the field of $p$-adic numbers

- $\mathfrak{H}$ the complex upper half plane

- $\zeta_{N}=e^{2 \pi i / N}$

- $i=\sqrt{-1}$

- $T_{N}^{*}$ a fundamental Thompson series (that is, a normalized Hauptmodule) for a genus zero group $\Gamma_{0}(N)^{*}$

- $R^{\times}$the group of units of a ring $R$

\section{Class fields by Shimura's canonical models}

Let $\Gamma$ be a Fuchsian group of the first kind. Then $X(\Gamma)=\Gamma \backslash \mathfrak{H}^{*}$ is a compact Riemann surface. Hence there exists a projective nonsingular algebraic curve $V_{\Gamma}$, defined over $\mathbb{C}$, biregularly isomorphic to $\Gamma \backslash \mathfrak{H}^{*}$. We specify a $\Gamma$-invariant holomorphic map $\varphi_{\Gamma}$ of $\mathfrak{H}^{*}$ to $V_{\Gamma}$ which gives a biregular isomorphism of $\Gamma \backslash \mathfrak{H}^{*}$ to $V_{\Gamma}$. In that situation, we call $\left(V_{\Gamma}, \varphi_{\Gamma}\right)$ a model of $\Gamma \backslash \mathfrak{H}^{*}$. For instance, if the genus of $\Gamma \backslash \mathfrak{H}^{*}$ is zero, then its function field $K(X(\Gamma))$ is equal to $\mathbb{C}\left(J^{\prime}\right)$ for some $J^{\prime} \in K(X(\Gamma))$ and hence the pair $\left(\mathbb{P}^{1}(\mathbb{C}), J^{\prime}\right)$ gives a model of $\Gamma \backslash \mathfrak{H}^{*}([4]$ Lemma 14).

Let $G_{\mathbb{A}}$ be the adelization of an algebraic group $G=G L_{2}$ defined over $\mathbb{Q}$. Put

$$
\begin{aligned}
G_{p} & =G L_{2}\left(\mathbb{Q}_{p}\right)(p: \text { a rational prime }), \\
G_{\infty} & =G L_{2}(\mathbb{R}), \\
G_{\infty+} & =\left\{x \in G_{\infty} \mid \operatorname{det}(x)>0\right\}, \\
G_{\mathbb{Q}_{+}} & =\left\{x \in G L_{2}(\mathbb{Q}) \mid \operatorname{det}(x)>0\right\}, \\
U & =\prod_{p} G L_{2}\left(\mathbb{Z}_{p}\right) \times G_{\infty+}, \\
G_{\mathbb{A}_{+}} & =U G_{\mathbb{Q}_{+}} .
\end{aligned}
$$

We define the topology of $G_{\mathbb{A}}$ by taking $U$ to be an open subgroup of $G_{\mathbb{A}}$. Let $K$ be an imaginary quadratic field as described in the introduction and $\xi_{\alpha}$ be an embedding of $K$ into $M_{2}(\mathbb{Q})$. We call $\xi_{\alpha}$ normalized if it is defined by $a\left(\begin{array}{c}\alpha \\ 1\end{array}\right)=\xi_{\alpha}(a)\left(\begin{array}{c}\alpha \\ 1\end{array}\right)$ for $a \in K$ where $\alpha$ is the fixed point of $\xi_{\alpha}\left(K^{\times}\right)\left(\subset G_{\mathbb{Q}_{+}}\right)$ in $\mathfrak{H}$. Observe that the embedding $\xi_{\alpha}$ defines a continuous homomorphism of $K_{\mathbb{A}}^{\times}$into $G_{\mathbb{A}+}$, which we denote again by $\xi_{\alpha}$. Indeed, $\xi_{\alpha}=\left(\xi_{\alpha, p}\right)$ is defined by $x_{p}\left(\begin{array}{c}\alpha \\ 1\end{array}\right)=\xi_{\alpha, p}\left(x_{p}\right)\left(\begin{array}{c}\alpha \\ 1\end{array}\right)$ for $x_{p} \in K \otimes_{\mathbb{Q}} \mathbb{Q}_{p}$ for all prime $p$. Here $G_{\mathbb{A}+}$ is the group $G_{0} G_{\infty+}$ with $G_{0}$ the non-archimedean part of $G_{\mathbb{A}}$ and $K_{\mathbb{A}}^{\times}$is the idele group of $K$. Let $\mathcal{Z}$ be the set of open subgroups $S$ of $G_{\mathbb{A}}$ containing $\mathbb{Q}^{\times} G_{\infty+}$ such that $S / \mathbb{Q}^{\times} G_{\infty+}$ is compact. For $S \in \mathcal{Z}$, we see that $\operatorname{det}(S)$ is open in $\mathbb{Q}_{\mathbb{A}}^{\times}$. Therefore the subgroup $\mathbb{Q}^{\times} \operatorname{det}(S)$ of $\mathbb{Q}_{\mathbb{A}}^{\times}$corresponds to a finite abelian extension of $\mathbb{Q}$, which we write $k_{S}$. Put $\Gamma_{S}=S \cap G_{\mathbb{Q}_{+}}$for $S \in \mathcal{Z}$. As is 
well known ([6] Proposition 6.27), $\Gamma_{S} / \mathbb{Q}^{\times}$is a Fuchsian group of the first kind commensurable with $P S L_{2}(\mathbb{Z})$. Let

$$
\begin{aligned}
U_{p}^{0} & =\left\{\left(\begin{array}{ll}
a & b \\
c & d
\end{array}\right) \in G L_{2}\left(\mathbb{Z}_{p}\right) \mid c \equiv 0 \bmod N \mathbb{Z}_{p}\right\}, \\
U^{0} & =\left\{x=\left(x_{p}\right) \in U \mid x_{p} \in U_{p}^{0} \text { for all finite } p\right\}, \\
U_{*}^{0} & =U^{0} \cup U^{0} \Phi(N), \\
\Phi(N) & =\left(x_{p}\right) \in G_{\mathbb{A}+} \text { with } x_{p}=\left(\begin{array}{cc}
0 & -1 \\
N & 0
\end{array}\right) .
\end{aligned}
$$

Then we have

Lemma 2.1. (i) $\mathbb{Q}^{\times} U_{*}^{0}, \mathbb{Q}^{\times} U^{0} \in \mathcal{Z}$,

(ii) $k_{S}=\mathbb{Q}$ for $S \in\left\{\mathbb{Q}^{\times} U_{*}^{0}, \mathbb{Q}^{\times} U^{0}\right\}$,

(iii) $\Gamma_{S}=\mathbb{Q}^{\times} \Gamma_{0}(N)^{*}$ (respectively, $\left.\mathbb{Q}^{\times} \Gamma_{0}(N)\right)$ if $S=\mathbb{Q}^{\times} U_{*}^{0}$ (respectively, $\left.\mathbb{Q}^{\times} U^{0}\right)$.

Proof. It is well known for $S=\mathbb{Q}^{\times} U^{0}$. Since $\mathbb{Q}^{\times} U_{*}^{0}=\mathbb{Q}^{\times} U^{0} \cup \mathbb{Q}^{\times} U^{0} \Phi(N)$ and $\mathbb{Q}^{\times} U^{0}$ is an open subgroup in $G_{A+}, \mathbb{Q}^{\times} U_{*}^{0}$ is also an open subgroup in $G_{A+}$. Observing the fact that $\mathbb{Q}^{\times} U^{0} / \mathbb{Q}^{\times} G_{\infty+}$ is compact, we obtain $\mathbb{Q}^{\times} U_{*}^{0} \in \mathcal{Z}$. As for (ii), we know that $\mathbb{Q}$ corresponds to the norm group $\mathbb{Q}^{\times} \mathbb{Q}_{\mathbb{A}}^{\times \infty}$ with $\mathbb{Q}_{\mathbb{A}}^{\times \infty}=\mathbb{R}^{\times} \prod_{p} \mathbb{Z}_{p}^{\times}$and $\operatorname{det}\left(U_{*}^{0}\right)=N \operatorname{det}\left(U^{0}\right) . \quad \operatorname{But} \operatorname{det}\left(U^{0}\right)=\mathbb{Q}_{\mathbb{A}}^{\times \infty}$, and hence by the class field theory $k_{S}=\mathbb{Q}$. Indeed, $\operatorname{det}\left(U^{0}\right)$ is contained in $\mathbb{Q}_{\mathbb{A}}^{\times \infty}$. Conversely, for any element $\left(\alpha_{p}\right) \in \mathbb{Q}_{\mathbb{A}}^{\times \infty}$, take $y_{p}=\left(\begin{array}{cc}1 & 0 \\ 0 & \alpha_{p}\end{array}\right)$; then $\left(y_{p}\right) \in U_{0}$ and $\operatorname{det}\left(y_{p}\right)=\left(\operatorname{det} y_{p}\right)=\left(\alpha_{p}\right)$. Lastly, we readily get that $\Gamma_{S}=\mathbb{Q}^{\times} U_{*}^{0} \cap G_{\mathbb{Q}+}=$ $\mathbb{Q}^{\times}\left(U_{*}^{0} \cap G_{\mathbb{Q}+}\right)=\mathbb{Q}^{\times} \Gamma_{0}(N)^{*}$.

For two complex numbers $\omega_{1}$ and $\omega_{2}$ such that $\omega_{1} / \omega_{2} \in \mathfrak{H}$, we have a lattice $L=\mathbb{Z} \omega_{1}+\mathbb{Z} \omega_{2}$ in $\mathbb{C}$. We then define a Fricke function

$$
f_{a}(z)=\frac{g_{2}\left(\omega_{1}, \omega_{2}\right) g_{3}\left(\omega_{1}, \omega_{2}\right)}{\Delta\left(\omega_{1}, \omega_{2}\right)} \mathfrak{p}\left(a\left(\begin{array}{c}
\omega_{1} \\
\omega_{2}
\end{array}\right) ; \omega_{1}, \omega_{2}\right) \quad\left(a \in \mathbb{Q}^{2} \backslash \mathbb{Z}^{2}\right),
$$

where

$$
\begin{aligned}
\mathfrak{p}\left(u ; \omega_{1}, \omega_{2}\right) & =u^{-2}+\sum_{w \in L \backslash 0}\left[(u-w)^{-2}-w^{-2}\right], \\
g_{2}\left(\omega_{1}, \omega_{2}\right) & =60 \sum_{w \in L \backslash 0} w^{-4}, \\
g_{3}\left(\omega_{1}, \omega_{2}\right) & =140 \sum_{w \in L \backslash 0} w^{-6} \text { and } \\
\Delta\left(\omega_{1}, \omega_{2}\right) & =g_{2}\left(\omega_{1}, \omega_{2}\right)^{3}-27 g_{3}\left(\omega_{1}, \omega_{2}\right)^{2} .
\end{aligned}
$$

Now let us put, for a positive integer $N$,

$$
\mathfrak{F}_{N}=\mathbb{Q}\left(j, f_{a} \mid a \in N^{-1} \mathbb{Z}^{2}, \notin \mathbb{Z}^{2}\right) \quad \text { and } \quad \mathfrak{F}=\bigcup_{N=1}^{\infty} \mathfrak{F}_{N},
$$

where $j$ is the elliptic modular function. Then $\mathfrak{F}$ is a Galois extension of $\mathfrak{F}_{1}$ and $\mathbb{C F}$ is the field of modular functions of all levels. 
Proposition 2.2. For any $u \in U$, one can define an element $\tau(u)$ of $\operatorname{Gal}\left(\mathfrak{F} / \mathfrak{F}_{1}\right)$ by $f_{a}^{\tau(u)}=f_{\text {au }}$ for all $a \in(\mathbb{Q} \backslash \mathbb{Z})^{2}$. Moreover, $\tau(u)$ has the following properties:

(1) The sequence $1 \rightarrow\{ \pm 1\} \cdot G_{\infty+} \rightarrow U \rightarrow \operatorname{Gal}\left(\mathfrak{F} / \mathfrak{F}_{1}\right) \rightarrow 1$ is exact,

(2) $\tau(u)=\left[\operatorname{det}(u)^{-1}, \mathbb{Q}\right]$ on $\mathbb{Q}_{a b}$,

(3) $h^{\tau(\gamma)}=h \circ \gamma$ for all $h \in \mathfrak{F}$ and $\gamma \in S L_{2}(\mathbb{Z})$.

Proof. See [6, Proposition 6.21].

Here, $[, \mathbb{Q}]$ is the reciprocity map and $\mathbb{Q}_{\mathrm{ab}}$ is the maximal abelian extension of $\mathbb{Q}$.

We shall now define a homomorphism $\tau: G_{\mathbb{A}_{+}} \longrightarrow$ Aut $(\mathfrak{F})$ as follows. Since $G_{\mathbb{A}_{+}}=U G_{\mathbb{Q}_{+}}$for $\beta \in G_{\mathbb{Q}_{+}}$, we define $\tau(\beta)$ by

$$
h^{\tau(\beta)}=h \circ \beta \quad \text { for all } h \in \mathfrak{F} .
$$

And, for $x=u \beta \in G_{\mathbb{A}_{+}}$with $u \in U$ and $\beta \in G_{\mathbb{Q}_{+}}$we put

$$
\tau(x)=\tau(u) \tau(\beta)
$$

so that $j^{\tau(x)}=j \circ \beta$ and $f_{a}^{\tau(x)}=f_{a u} \circ \beta$. Indeed, the map $\tau$ is defined independently of the choice of $u$ and $\beta$ by virtue of Proposition 2.2.

Next, for $S=\mathbb{Q}^{\times} U_{*}^{0}$ or $\mathbb{Q}^{\times} U^{0}$ we can find a model $\left(V_{\Gamma_{S}}, \varphi_{\Gamma_{S}}\right)$ of the curve $\Gamma_{S} \backslash \mathfrak{H}^{*}$, which is characterized by the following properties:

(i) $V_{S}$ is defined over $k_{S}=\mathbb{Q}$ (Lemma 2.1),

(ii) $\mathfrak{F}_{S}=\left\{f \circ \varphi_{\Gamma_{S}} \mid f \in \mathbb{Q}\left(V_{\Gamma_{S}}\right)\right\}$

where $\mathfrak{F}_{S}=\left\{h \in \mathfrak{F} \mid h^{\tau(x)}=h\right.$ for all $\left.x \in S\right\}$ and $\mathbb{Q}\left(V_{\Gamma_{S}}\right)$ denotes the field of functions on $V_{\Gamma_{S}}$ rational over $\mathbb{Q}$.

Example 2.3. Let $T_{N}^{*}$ be a fundamental Thompson series for a genus zero group $\Gamma_{0}(N)^{*}$ and $S=\mathbb{Q}^{\times} U_{*}^{0}$. Then $\mathfrak{F}_{S}=\mathbb{Q}\left(T_{N}^{*}\right)$ because $\mathbb{C}$ is linearly disjoint with $\mathfrak{F}_{S}$ over $k_{S}(=\mathbb{Q})$.

Theorem 2.4. Let $\alpha$ be a root in $\mathfrak{H}$ of a primitive integral equation $a z^{2}+$ $b z+c=0$ with $a>0$ such that $b^{2}-4 a c=m^{2} d_{K}(<0)$. Put $K=\mathbb{Q}(\alpha)$ and $\mathcal{O}(=\mathbb{Z}[a \alpha])$ be an order in $K$ of discriminant $m^{2} d_{K}$, where $d_{K}$ is the discriminant of $K$.

(1) If $N \geq 1, S=\mathbb{Q}^{\times} U^{0}$ and $\left(V_{\Gamma_{S}}, \varphi_{\Gamma_{S}}\right)$ is Shimura's canonical model as in the above, then $\varphi_{\Gamma_{S}}(\alpha)$ generates the ring class field of an order $\mathcal{O}^{\prime}$ of discriminant $f^{2} d_{K}$ where the conductor $f$ of $\mathcal{O}^{\prime}$ is $m N /(a, N)$.

(2) Assume that $(a, b, N) \neq N$ or $\left(\frac{a}{N}, N\right) \neq 1$ for $N \geq 2$. If $S=\mathbb{Q}^{\times} U_{*}^{0}$ and $\left(V_{\Gamma_{S}}, \varphi_{\Gamma_{S}}\right)$ is a model, then $\varphi_{\Gamma_{S}}(\alpha)$ generates the ring class field of an order $\mathcal{O}^{\prime}$ of discriminant $f^{2} d_{K}$ where its conductor $f$ equals $m N /(a, N)$. 
Proof. Let $L$ be a lattice $=\mathbb{Z} N \alpha+\mathbb{Z}$. Then $\mathcal{O}^{\prime}=\mathbb{Z}+\frac{m N}{(a, N)} \mathcal{O}_{K}$ is an order of $L$ in $K([5]$ Ch.8). Let us consider the commutative diagram

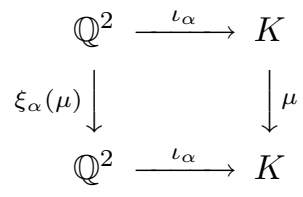

where $\iota_{\alpha}(x, y)=x \alpha+y$ and $\iota_{\alpha}\left((x, y) \xi_{\alpha}(\mu)\right)=\mu \iota_{\alpha}(x, y)$.

We may consider the mapping $\iota_{\alpha}$ (respectively, $\xi_{\alpha}: K^{\times} \rightarrow G L_{2}(\mathbb{Q})$ ) as an isomorphism of affine varieties (respectively, a homomorphism of algebraic groups) over $\mathbb{Q}$. Thus, taking the $\mathbb{Q}_{\mathbb{A}}$-valued points, we have the following commutative diagram

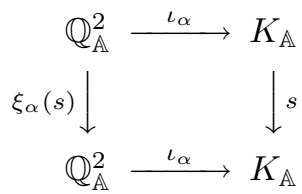

for any idele $s \in K_{\mathbb{A}}^{\times}$.

Let $s=s_{\infty} s_{0} \in K_{\mathbb{A}}^{\times}$with infinite part $s_{\infty}$ and finite part $s_{0}=\left(s_{p}\right)_{p}$ of $s$. We then have the following statements:

$$
\begin{aligned}
\xi_{\alpha}(s) \in U \quad & \text { if and only if } \xi_{\alpha, p}\left(s_{p}\right) \in G L_{2}\left(\mathbb{Z}_{p}\right) \text { for all finite } p . \\
& \text { if and only if } \xi_{\alpha}\left(s_{0}\right) \in \prod_{p} G L_{2}\left(\mathbb{Z}_{p}\right) . \\
& \text { if and only if } \xi_{\alpha}\left(s_{0}\right) \text { induces an automorphism of }\left(\prod_{p} \mathbb{Z}_{p}\right)^{2} .
\end{aligned}
$$

if and only if the multiplication by $s_{0}$ induces an

automorphism of $(\mathbb{Z} \alpha+\mathbb{Z}) \otimes_{\mathbb{Z}} \hat{\mathbb{Z}}$

(because $\left.\iota_{\alpha}\left[\left(\prod_{p} \mathbb{Z}_{p}\right)^{2}\right]=(\mathbb{Z} \alpha+\mathbb{Z}) \otimes_{\mathbb{Z}} \hat{\mathbb{Z}}\right)$.

if and only if $s_{0} \in\left(\mathcal{O}_{1} \otimes_{\mathbb{Z}} \hat{\mathbb{Z}}\right)^{\times}$

where $\mathcal{O}_{1}$ is the order of $\mathbb{Z} \alpha+\mathbb{Z}$ in $K$.

Similarly, when $\xi_{\alpha}(s) \in U$, we have that

$$
\begin{aligned}
\xi_{\alpha}(s) \in U^{0} & \text { if and only if } \xi_{\alpha}\left(s_{0}\right) \text { induces an automorphism of } \\
& \left(N \prod_{p} \mathbb{Z}_{p}\right) \oplus \prod_{p} \mathbb{Z}_{p} . \\
& \text { if and only if the multiplication by } s_{0} \text { induces an } \\
& \text { automorphism of } L \otimes_{\mathbb{Z}} \hat{\mathbb{Z}} . \\
& \text { if and only if } s_{0} \in\left(\mathcal{O}^{\prime} \otimes_{\mathbb{Z}} \hat{\mathbb{Z}}\right)^{\times} .
\end{aligned}
$$


Then the assertion (1) follows from this, Lemma 2.1 and [6, Proposition 6.33] that $K \cdot k_{S}\left(\left(\varphi_{\Gamma_{S}}(\alpha)\right)=K\left(\varphi_{\Gamma_{S}}(\alpha)\right)\right.$ is the ring class field of $\mathcal{O}^{\prime}$.

In order to verify (2), we have only to show that $\xi_{\alpha}\left(K_{\mathbb{A}}^{\times}\right) \cap U^{0} \Phi(N)$ is an empty set under our assumptions. For $x_{p} \in K_{p}^{\times}$, let

$$
\xi_{\alpha, p}\left(x_{p}\right)=\left(\begin{array}{cc}
a_{p} & b_{p} \\
c_{p} & d_{p}
\end{array}\right)
$$

with $a_{p}, b_{p}, c_{p}, d_{p} \in \mathbb{Q}_{p}$.

Then

$$
\left(\begin{array}{ll}
a_{p} & b_{p} \\
c_{p} & d_{p}
\end{array}\right)\left(\begin{array}{c}
\alpha \\
1
\end{array}\right)=x_{p}\left(\begin{array}{c}
\alpha \\
1
\end{array}\right)
$$

implies

$$
\xi_{\alpha, p}\left(x_{p}\right)=\left(\begin{array}{cc}
d_{p}-\frac{b}{a} c_{p} & \frac{-c}{a} c_{p} \\
c_{p} & d_{p}
\end{array}\right) .
$$

Now suppose that $\xi_{\alpha}\left(\left(x_{p}\right)\right)=\left(\xi_{\alpha, p}\left(x_{p}\right)\right)_{p} \in U^{0} \Phi(N)$ for some $\left(x_{p}\right)_{p} \in K_{\mathbb{A}}^{\times}$. For each finite prime $p$, we can write

$$
\left(\begin{array}{cc}
d_{p}-\frac{b}{a} c_{p} & \frac{-c}{a} c_{p} \\
c_{p} & d_{p}
\end{array}\right)=\left(\begin{array}{c}
N \beta_{p}-\alpha_{p} \\
N w_{p}-\gamma_{p}
\end{array}\right)
$$

with some $\left(\begin{array}{cc}\alpha_{p} & \beta_{p} \\ \gamma_{p} & w_{p}\end{array}\right) \in G L_{2}\left(\mathbb{Z}_{p}\right)$ satisfying $\gamma_{p} \equiv 0 \bmod N \mathbb{Z}_{p}$.

Then $\alpha_{p} w_{p}-\gamma_{p} \beta_{p} \in \mathbb{Z}_{p}^{\times}$says that $\alpha_{p}, w_{p} \in \mathbb{Z}_{p}^{\times}$for all prime $p \mid N$. Notice that $c_{p}=N w_{p}$ and $d_{p}=-\gamma_{p} \in \mathbb{Z}_{p}$ for all prime $p \mid N$. Since $\frac{c}{a} c_{p}=\frac{c}{a} N w_{p}=\alpha_{p} \in \mathbb{Z}_{p}^{\times}$ for all prime $p \mid N,(a, N)=N$. Moreover, $\frac{b}{a} w_{p} \in \mathbb{Z}_{p}$ and hence $(b, N)=N$ because $d_{p}-\frac{b}{a} c_{p}=-\gamma_{p}-\frac{b}{a} N w_{p}=N \beta_{p}$ and $N \mid \gamma_{P}$ for all prime $p \mid N$. If $\left(\frac{a}{N}, N\right) \neq 1$, then we can take a prime factor $p$ of $\left(\frac{a}{N}, N\right)$. Our factor $p$ divides $c$ because $\frac{c}{a} N w_{p}=\alpha_{p} \in \mathbb{Z}_{p}^{\times}$and $\frac{c}{a} N \in \mathbb{Z}_{p}^{\times}$. Therefore, $p$ divides $(a, b, c)$, which is a contradiction.

Corollary 2.5. Notations and assumptions being the same as in Theorem 2.4, we further suppose that $(a, b, N) \neq N$ or $\left(\frac{a}{N}, N\right) \neq 1$ for $N \geq 2$. Then $T_{N}^{*}(\alpha)$ generates the ring class field of an order $\mathcal{O}^{\prime}$ of discriminant $f^{2} d_{K}$ whose conductor $f$ is $m N /(a, N)$.

Proof. It is immediate from Lemma 2.1 and Theorem 2.4(2).

Note that this corollary can also be proved by Chen-Yui's method.

\section{References}

[1] I. Chen and N. Yui, Groups, Difference Sets and the Monster (OSU Math. Research Inst. Publication 4), 255-326, Walter de Gruyter \& Co. 1995.

[2] J. H. Conway and S. P. Norton, Monstrous moonshine, Bull. London Math. Soc. 11 (1979), no. 3, 308-339.

[3] H. Helling, Note über das Geschlecht gewisser arithmetischer Gruppen, Math. Ann. 205 (1973), 173-179.

[4] C. H. Kim and J. K. Koo, Arithmetic of the modular function $j_{1,4}$, Acta Arith. 84 (1998), no. $2,129-143$.

[5] S. Lang, Elliptic Functions, Springer-Verlag, 1987. 
[6] G. Shimura, Introduction to the Arithmetic Theory of Automorphic Functions, Publications of the Mathematical Society of Japan, No. 11. Iwanami Shoten, Publishers, Tokyo; Princeton University Press, Princeton, N.J., 1971.

SoYoung Choi

Department of Mathematics Education

DONGGUK UNIVERSITY

GyeongJu 780-714, KoreA

E-mail address: young@dongguk.ac.kr

JA KYUNG KoO

Korea Advanced Institute of Science and Technology

Department of Mathematical Sciences

TAEJON 305-701, KorEA

E-mail address: jkkoo@math.kaist.ac.kr 